\title{
Interacción en la improvisación jazzística: El análisis de los aspectos rítmicos en el ciclo de percepción-acción*
}

\author{
JOAQUÍN B. PÉREZ, ISABEL C. MARTÍNEZ ${ }^{* *}$
}

\begin{abstract}
Resumen
Este trabajo aborda el estudio de la interacción rítmica en la improvisación musical jazzística. Desde la perspectiva de la cognición musical corporeizada se describe a la improvisación como una forma de interacción ecológica del músico con su entorno (Johnson 2007; Clarke, 2005). Se recurre para su análisis, al concepto de ciclo de percepción-acción (Leman, 2008) y se define al mismo en tres fases que incluyen: (i) la acción realizada en la ejecución; (ii) la percepción corporal y auditiva del resultado de la propia acción y/o la de otros ejecutantes; y (iii) la reconfiguración de la acción en curso durante la performance. Para el estudio de estas tres fases se realiza un experimento en el que un grupo de músicos improvisa sobre una base MIDI de jazz-blues. El modo en el que los improvisadores se adaptan y posteriormente ajustan rítmicamente con la base en un nivel micro-temporal se describe recurriendo al marco teórico y metodológico de la teoría del entrainment (Clayton, 2005).
\end{abstract}

Palabras-clave: improvisación, cognición corporeizada, entrainment Interaction in jazz improvisation: Analysis of the rhythmic aspects in the
perception-action cycle

Abstract

This paper focuses on the study of rhythmic interaction during music improvisation in jazz. Based on the theory of embodied music cognition, music improvisation is characterized as an interactive ecological exchange between the music and the environment (Johnson 2007; Clarke, 2005). The concept of perception-action cycle (Leman 2008) is applied to the analysis of music improvisation. Three stages are defined in this cycle: (i) improviser's realized action during performance; (ii) embodied and aural perception of the result of improviser's own performance; and (iii) action reconfiguration on course during performance. An experiment was run to study the perception-action cycle. A group of improvisers improvised along a jazz-blues MIDI base. The way musicians adapt their microtiming rhythmic adjustment to changes in the temporal organization of the stimuli is described according to the theoretic and methodological framework of entrainment theory (Clayton, 2005).

Keywords: improvisation, embodied cognition, entrainment

\footnotetext{
* Este artículo es una versíon ampliada y revisada del documento presentado en el XI SIMCAM - Simpósio Internacional de Cognição e Artes Musicais, realizado por la Universidad Federal de Goiás, en Pirenópolis/GO, Brasil, mayo 26-29, 2015.

** LEEM (Laboratorio para el Estudio de la Experiencia Musical)

Facultad de Bellas Artes. Universidad Nacional de La Plata

Email: joaq81@hotmail.com; isabelmartinez@fba.unlp.edu.ar
} 


\section{Introducción}

La práctica de la improvisación musical en el jazz - sea ésta el resultado de las acciones individuales o grupales-es una manifestación musical que compromete la realización de un complejo performativo de índole multimodal. En este trabajo nos enfocamos específicamente en la actividad rítmico-temporal que el improvisador desarrolla durante la performance.

Estudios etnomusicológicos se han interesado en el análisis de la práctica intersubjetiva que tiene lugar durante la actuación de los músicos en el interior de un grupo musical (Berliner, 1994; Monson, 1998). La interacción en un conjunto de jazz con una instrumentación de base estándar', se configura y restringe frecuentemente en el marco de las convenciones particulares del estilo. Berliner describe en Thinking in Jazz, obra paradigmática de la literatura etnomusicológica, el modo en el que la base de acompañamiento y el solista se condicionan mutuamente a partir de la imitación rítmica y melódica. En el trabajo citado se presenta un exhaustivo análisis de un conjunto amplio de patterns rítmicos y melódicos intra-opus que se repiten en las improvisaciones del conjunto de jazz. Por otra parte, tanto Berliner como Monson identifican la presencia de una modalidad especial de interacción que se manifiesta en el nivel micro-temporal del ritmo, a la que los músicos describen utilizando términos como "sensación de groove". El concepto groove, de uso común en el estilo, describe una sensación especial de ajuste o entonamiento temporal grupal y de coherencia rítmica.

A diferencia de la construcción del discurso improvisatorio en base a la imitación rítmica o melódica, la microorganización temporal del ritmo en la performance no puede analizarse en profundidad utilizando las herramientas tradicionales de la etnomusicología, tales como la transcripción o la entrevista. El estudio del ritmo en un nivel micro-temporal en el jazz se hace posible mediante el uso de herramientas de software sofisticadas para el análisis del audio digital. En el campo de la musicología sistemática, Mark Doffman (2012) aborda el estudio del groove y de la intersubjetividad en el jazz enmarcando estos problemas en la teoría del entrainment. La medición detallada de las desviaciones de timing en grabaciones de improvisaciones al interior de un grupo musical provee datos precisos y objetivos, susceptibles de ser comparados con las descripciones verbales de los improvisadores acerca del groove La acción musical compartida en la improvisación jazzística es experimentada

\footnotetext{
${ }^{1}$ En el jazz, una base estándar de acompañamiento se compone de: (i) una figura rítmica de acompañamiento o bajo caminado en negras a cargo del contrabajo o bajo eléctrico; (ii) un ritmo similar o subdividido a cargo de la batería sobre uno de los platillos (ryde); (iii) un acompañamiento denominado coniping a cargo del piano. El comping configura un acompañamiento rítmico sobre acordes plaqué. Es sobre esta base estándar que por lo general improvisa el solista.
} 
como groove por parte de los improvisadores en un nivel pre-conciente. El microanálisis permite describir así las diferentes formas de construir el groove al interior de la base de acompañamiento y entre la base y el solista (Doffman, 2012).

La investigación en cognición musical corporeizada, por su parte, también ha propuesto el estudio de la performance musical como una forma de interacción ecológica del músico con su entorno (Johnson 2007; Leman 2008; Clarke, 2005). Llevada al terreno de la improvisación musical, esta interacción podría definirse como un proceso de continua adaptación en el que el ejecutante organiza los eventos sonoros que percibe mientras toca, en un ciclo experiencial único en el que percepción y acción se separan solo a los fines del análisis.

En el presente trabajo se describen los resultados de un estudio del ciclo de percepción-acción en la improvisación. Para tal fin se postula que durante la práctica de la improvisación se desarrolla un tipo de "conciencia en acto", por medio de la audición-en-acción de la propia performance y de la performance de otros músicos dentro del conjunto de jazz. Se investigan los aspectos rítmicos y micro-temporales de su funcionamiento. En la primera parte del artículo se caracteriza el ciclo de percepción-acción dentro de los estudios de la cognición musical corporeizada y se describe su vinculación con las teorías de la expectación y el entrainment. En la segunda parte se reportan los resultados de un experimento realizado para estudiar su funcionamiento en un contexto de laboratorio durante la práctica de la performance improvisada. Por último se discuten sus alcances para una caracterización de la práctica de la improvisación musical como una ontología orientada por la percepción en acción.

\section{Parte 1}

\subsection{El ciclo de percepción-acción}

En términos cognitivos la ejecución improvisada puede ser caracterizada como un proceso cíclico y temporal en el que el improvisador se adapta a los rasgos estructurales y a los acontecimientos novedosos que tienen lugar momento a momento durante la performance. Para explicar este proceso Leman (2008) propone un modelo dinámico que llama de acción-reacción. Referido más específicamente a la práctica improvisatoria y sobre la base del modelo de Leman, en trabajos anteriores (Pérez \& Martínez, 2012) se propuso un ciclo de percepción-acción para modelizar el funcionamiento temporal del proceso improvisatorio. El ciclo de percepción-acción se desarrolla en tres fases. Estas incluyen: (i) la acción realizada en la ejecución 
de manera automática o reflexiva; (ii) la percepción corporal y auditiva del resultado de la propia acción y/o la de otros ejecutantes; y (iii) la reconfiguración de la acción en curso durante la performance (Figura 1).

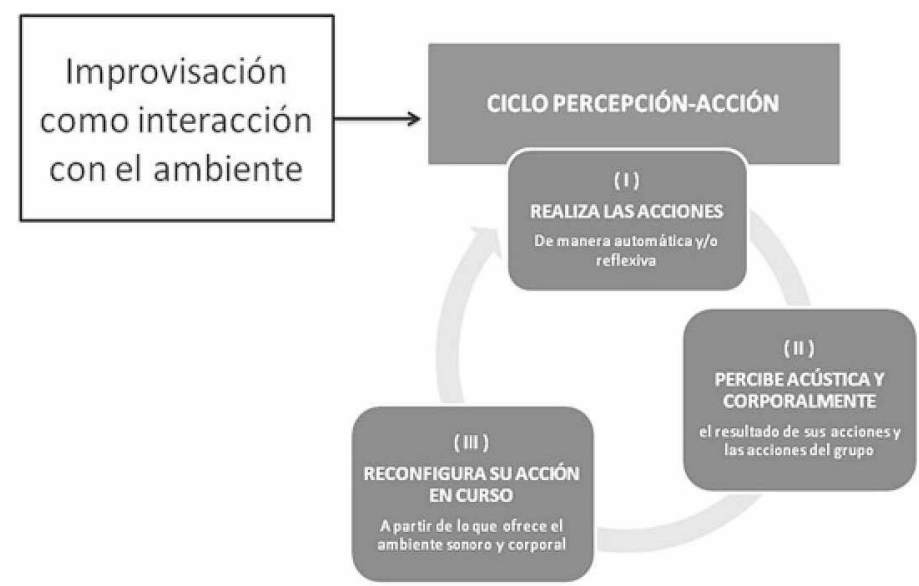

Figura 1. El Ciclo de Percepción-Acción en la improvisación en tres fases: (i) acción; (ii) percepción y (iii) reconfiguración de la acción.

Cabe aclarar que las fases del ciclo de percepción-acción que se postulan constituyen solo categorías para el estudio teórico del fenómeno, por lo que podemos considerar que según la ventana temporal que utilicemos para el análisis, dichas fases no solo se suceden sino que además pueden yuxtaponerse, siendo sus límites difusos. El improvisador no deja de percibir mientras toca, ni deja de tocar cuando reconfigura su acción; para acercarnos a la percepción-acción como un todo debemos abordarla a partir de un análisis dinámico que estudie su funcionamiento en el devenir de la temporalidad.

\subsection{La ruptura de las expectativas y la reconfiguración de la acción}

A partir de la caracterización del ciclo de percepción-acción en la improvisación surgen las siguientes preguntas: ¿cómo se reconfigura la acción en base a lo percibido? ¿Cómo se percibe la actividad que se realiza en el devenir mismo de la performance? Con el fin de responderlas se diseña un experimento a partir del cual se observa minuciosamente el modo en el que el improvisador actúa a partir de la percepción de ciertos cambios introducidos en la base de acompañamiento que rompen con sus expectativas musicales. Se introduce de esta manera el concepto de expectativas (Meyer, 1956; Huron, 2006) como un dispositivo mediante el cual sería posible evaluar analíticamente el vínculo entre lo percibido y la actividad que se realiza en la improvisación musical. 
Los tipos de respuesta a un estímulo han sido analizados por D. Huron (2006) en su modelo teórico sobre la expectación. Según este autor los organismos en general y los seres humanos en particular responden mejor a los eventos esperados porque pueden explotar las oportunidades que brinda la anticipación. Por el contrario, los eventos inesperados, donde la expectativa no es confirmada, producen diferentes tipos de respuestas: algunas son respuestas corporales inmediatas y automáticas, a las que podemos llamar reacción; otras respuestas pueden estar mediatizadas por el pensamiento consciente: son respuestas evaluadas. La ruptura de las expectativas del improvisador daría lugar a estos diferentes tipos de respuesta con diferentes grados de automatismo y/o control. Las categorías de reacción como respuesta inmediata y de evaluación en relación a las respuestas posteriores, que involucran acciones performativas o la interrupción de la acción, se utilizan en el presente trabajo como categorías de análisis del tipo de respuesta del improvisador ante la ruptura de sus expectativas hasta lograr el ajuste a la nueva pauta.

\subsection{El entrainment como metodología de análisis para la interacción rítmica}

Para poder estudiar el ciclo de percepción-acción se necesita observar el modo en el que los improvisadores reconfiguran temporalmente la acción durante la performance. En trabajos anteriores se realizaron análisis sobre el modo en el que un grupo de músicos reconfiguraba su acción en relación a los aspectos armónicos en la improvisación (Pérez, 2013). En este trabajo el interés está colocado en los aspectos de la temporalidad y el ritmo durante la interacción del ejecutante con el conjunto. La interacción rítmica puede ser operativizada recurriendo al marco teórico y a las categorías emergentes de la teoría del entrainment (Clayton, 2005). El modelo del entrainment describe el proceso a partir del cual dos sistemas rítmicos independientes u osciladores interactúan y se ajustan hasta acoplarse en una fase común. Si bien el entrainment musical implica en principio la intervención de dos o más ejecutantes que interactúan en la performance y se adaptan mutuamente, también se ha considerado la posibilidad de estudiar la interacción entre sistemas mecánicos y biológicos e incluso se ha planteado la función del entrainment interno, es decir entre nuestro propio movimiento y el modo en el que lo percibimos (Jones, 2000, como se cita en London, 2012). Aplicado al ámbito de la música, el entrainment es útil para describir los procesos cognitivos vinculados a la regulación temporal en la ejecución (timing). Doffman (2008) aborda el problema específico del groove en el jazz en el marco de un estudio de entrainment intra-grupo, en el cual mide y compara los onsets de sonidos particulares en archivos de audio digital. En 
este marco, el autor considera al groove como un tipo de relación dinámica en la que existe un grado permitido de ajuste o desajuste entre los beats de cada uno de los integrantes de un grupo. Desde esta perspectiva, entrar y permanecer en groove implicaría una conducta adaptativa, consciente o inconsciente en el transcurso de la interacción ajustando continuamente la performance a los límites permitidos de desvío de la pauta temporal.

\section{Parte 2}

\subsection{El diseño del experimento}

Para estudiar los ciclos de percepción-acción que intervienen en la configuración temporal de la improvisación se diseñó un experimento de laboratorio en el que un grupo de nueve improvisadores interactúan con una base MIDI. La base simula un ensamble de jazz y se compuso especialmente para indagar el modo en el que los músicos se adaptan a ciertos eventos inesperados, que rompen sus expectativas en el transcurso de la improvisación (Huron, 2006). Las rupturas introducidas constituyen una versión exagerada de las variaciones que se dan en la práctica musical real. Se realizó la comparación de los intervalos inter beat o $\mathrm{IBI}^{2}$, correspondientes a la base MIDI con aquellos correspondientes a las ejecuciones de los improvisadores en sus performances. El estudio del entrainment en los momentos donde se producen los cambios de tempo y se rompen las expectativas brindó importantes datos acerca del modo en el que el improvisador reconfigura la acción. En los apartados que siguen se describen las características del estudio realizado y se reporta el análisis de los resultados.

\subsection{Objetivo}

El objetivo de este estudio fue caracterizar los ciclos de percepción-acción que tienen lugar en la performance improvisada en un blues de jazz, a partir de ciertas rupturas con las expectativas que fueron provocadas introduciendo cambios en los parámetros de una base MIDI diseñada para tal propósito. Las respuestas de los improvisadores en lo que respecta a los cambios en los parámetros vinculados al ritmo, son analizadas con el fin de describir los modos que adquiere la conducta adaptativa de los ejecutantes al interactuar con la base.

${ }^{2}$ El intervalo inter beat o en ingles inter beat interval (IBI), es una categoría utilizada en acústica para definir el tiempo entre los onset. de los sonidos que se corresponden con el beat. 


\subsection{Método}

Se le propuso a un grupo de improvisadores tocar sobre una base MIDI que simula un ensamble de jazz en un típico esquema armónico-formal de blues. La interacción ejecutante-base es de tipo unidireccional; esto es, que el improvisador se adapta constantemente a la base grabada, en tanto que esta última solo simula interactuar con el improvisador a partir de la introducción de cambios temporales no esperados por el ejecutante. Las rupturas introducidas constituyen una versión exagerada de las variaciones que en diferentes aspectos se dan en la práctica musical real de una jam sesión o performance en vivo.

Estímulos: Una Base MIDI, en instrumental de walking bass, platillo ride y acompañamiento de piano, sintetizada especialmente para el estudio mediante los software Finale 2011 y Nuendo 4. La base se organiza a partir de un blues de 12 compases en Sib. Esta estructura se repite cuatro veces. La base presenta una serie de cambios abruptos de tempo (120 bpm-171 bpm). Al momento temporal puntual donde se produce cada cambio se lo denominó onset del cambio (OC).

Sujetos: nueve improvisadores (varones) (saxofón, clarinete, trompeta y trombón) con más de 10 años de experiencia y una edad promedio de 27 años, en la práctica de la improvisación vinculada al jazz.

Aparatos: Computadora, micrófono, auriculares, cámaras de video. Software Finale, Nuendo y Sonic Visualizer.

Procedimiento: Los improvisadores fueron grabados y filmados durante la performance en sincronía con la base. Las bases fueron reproducidas desde la PC por auriculares, la grabación se realizó en un programa multipistas (Nuendo 4) en un canal independiente de la base. La ejecución fue registrada al mismo tiempo por dos cámaras de video. Se les solicitó a los músicos considerar a la base como un intérprete o ejecutante real, no detenerse y usar en la improvisación un lenguaje moderno estándar de jazz. Se les mencionó que se trataba de un blues en $\mathrm{B}_{b}$ ( $\mathrm{Si}$ bemol), se les proporcionó un cifrado y se les solicitó que toquen lo más continuo posible, apoyados en el nivel métrico de la corchea. Luego de cada improvisación se realizó una breve entrevista semiestructurada con el fin de recabar información de primera persona acerca de experiencia de cada músico en relación a los cambios introducidos en la performance improvisada frente a los cambios acontecidos. 


\subsection{Resultados}

El microanálisis se realizó sobre un segmento de audio con una duración total de 24 seg aproximadamente. La metodología utilizada para la medición consistió en tomar los archivos de audio correspondientes a los nueve sujetos y realizar el siguiente procedimiento:

(i) - Recortar en Nuendo el segmento de 24 seg que incluye el cambio de tempo. El segmento de $24 \mathrm{seg}$ consta de un segmento inicial de seis compases ( 24 beats $-12 \mathrm{seg}$ ) que discurren a $120 \mathrm{bpm}$ hasta el onset del cambio (OC) y de un segmento de 8 compases (32 beats-11,2 seg) que discurren a $171 \mathrm{bpm}$ después de producido el OC.

(ii) - Aplicar la herramienta de detección del beat QM Vamp Plugin set - Tempo and Beat Tracker ${ }^{3}$ del Sonic Visualizer, a cada uno de los audios en cuestión.

(iii) - Corregir manualmente y a partir de la escucha seleccionando solo aquellos onsets que se interpretan como tactus y eliminando aquellos que no lo son. Puede observarse la captura de pantalla del Sonic Visualiser en la Figura 2.

(iv) - Medir todos los intervalos inter beat (IBI), que corresponden a los intervalos temporales entre los onset correspondientes a los beats identificados y exportar la información numérica, para luego trabajar con ella en una planilla de cálculo (Excel 2010).

(v)-Ordenar y alinear los datos de los nueve sujetos en relación a los 55 beats correspondientes al segmento de la ventana; los momentos en los que el improvisador no toca figuran como datos en blanco, estas detenciones pueden observarse en los gráficos que se presentan en los próximos apartados.

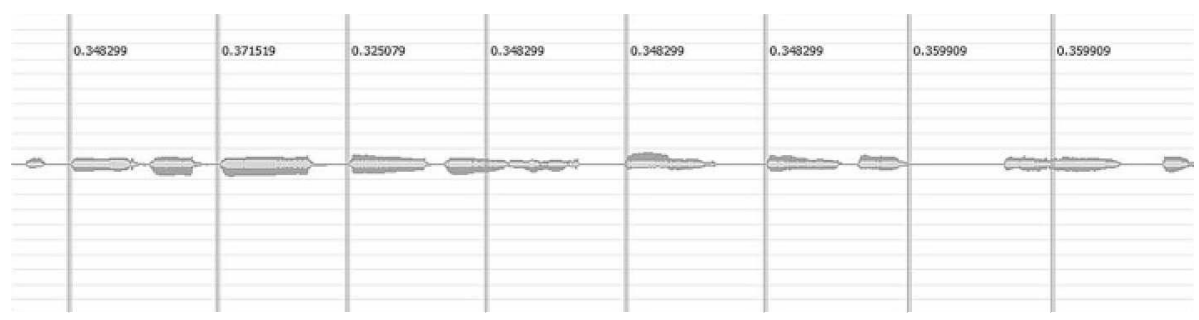

Figura 2. Captura de pantalla del Sonic Visualiser. Medición del IBI en el audio correspondiente al S3.

(vi) - A partir del análisis y la comparación de los datos numéricos se agrupó a los sujetos según tuvieran una conducta similar a partir del OC. En todos los casos un IBI=0,5 se corresponde con un tempo de $120 \mathrm{bmp}$; mientras que un IBI=0,35 aprox. se corresponde con 171 $\mathrm{bpm}$. Se detectaron conductas similares con respecto al modo en que

${ }^{3}$ QM Vamp Plugin set - Tempo and Beat Tracker es un software gratuito desarrollado por el Centro de Música Digital de Queen Mary, en University of London. Descargado el 20-4-2014 en http://vamp-plugins.org/plugin-doc/qm-vamp-plugins.html. 
los músicos se ajustan al nuevo tempo: interrumpiendo su acción, modificándola inmediatamente o generando grandes variaciones de IBI antes del ajuste.

\subsection{Análisis de resultados}

\section{Análisis caso por caso}

Se presentan a continuación los resultados para cada uno de los sujetos:

S5: Es el único caso en el que se produce un ajuste casi automático. Si bien se miden variaciones considerables del IBI antes del OC, luego del mismo el sujeto se adapta rápidamente ( 2 a 3 beats) sin interrumpir la ejecución.

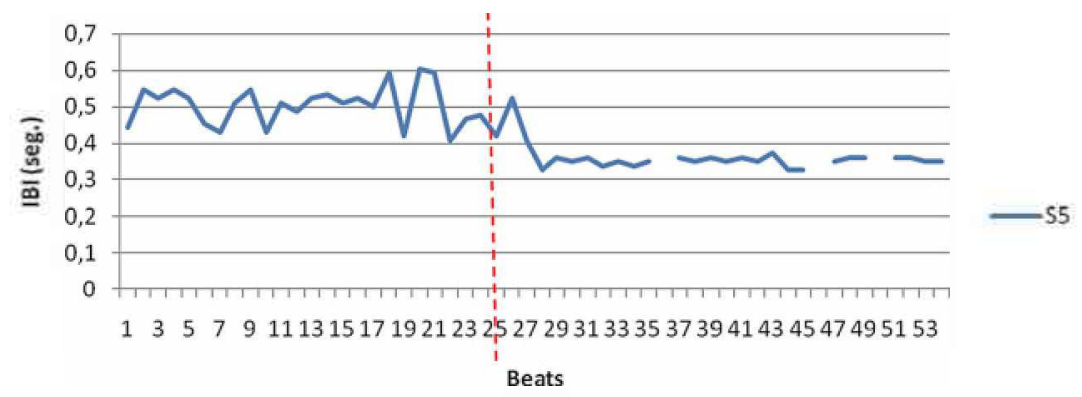

Figura 3. Medición del IBI en el S5, en el momento donde se produce el OC (120 bpm a $171 \mathrm{bpm}$ ) (línea de puntos roja).

S1 y S4: Los sujetos interrumpen su acción, evalúan y luego de un período donde se asume que S1 y S4 focalizan su atención en la base, se ajustan. En el caso de S4 la variación del IBI es mucho mayor, incluso cuando se considera que la performance está casi ajustada (Figura 4).

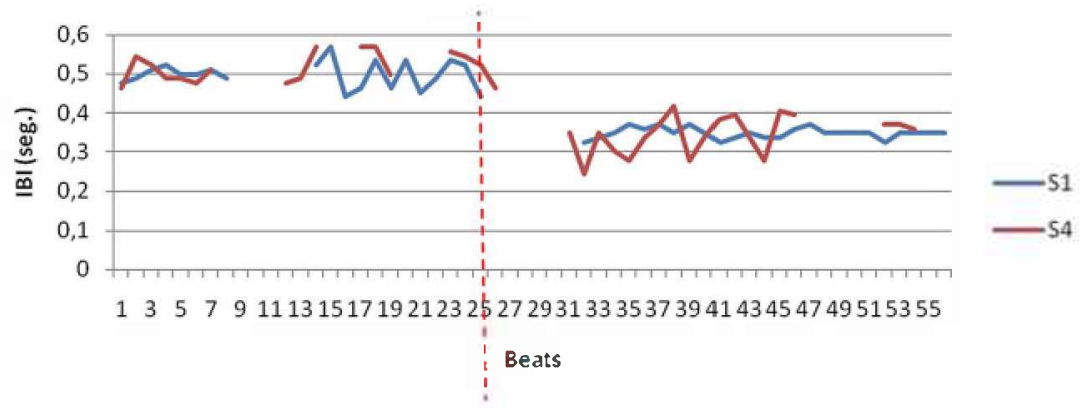

Figura 4. Medición del IBI en S1 y S4, la línea de puntos roja indica el OC $(120-171 \mathrm{bpm})$

S3-S7 y S3-S2: Los sujetos tienen una demora en el ajuste de entre 10 y 12 beats después del cambio de tempo. En el caso de S2 y S6, las variaciones del IBI previas al OC son considerables; luego del mismo se producen menos variaciones, una interrupción y posteriormente el ajuste con el beat de la base (Figura 5). En el caso de S3 y S7 la performance es más fragmentada, se producen varios silencios en los que, se asume, el improvisador evalúa lo percibido (Figura 6). 


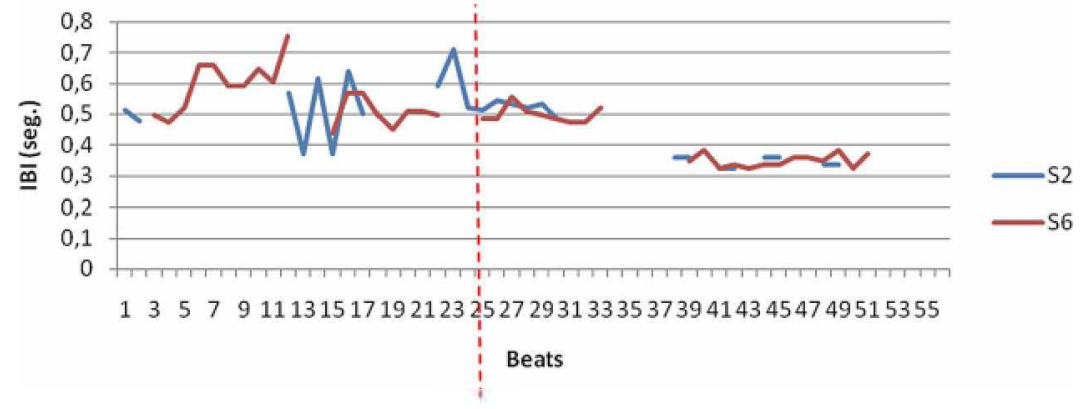

Figura 5. Medición del IBI en S2 y S6, la línea de puntos roja indica el OC (120-171 bpm)

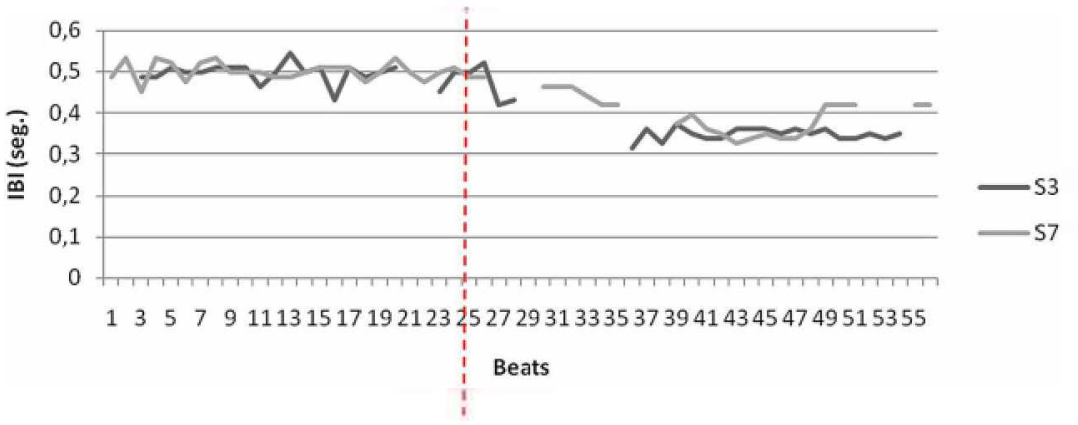

Figura 6. Medición del IBI en S3 y S7, la línea de puntos roja indica el OC (120-171 bpm)

S8 y S9: En la temporalidad de la performance se registra y visualiza una considerable variación en el IBI, la que se interpreta como un período de reacción al cambio; posteriormente se interrumpe la acción hasta que por fin ocurre la adaptación al nuevo tempo.

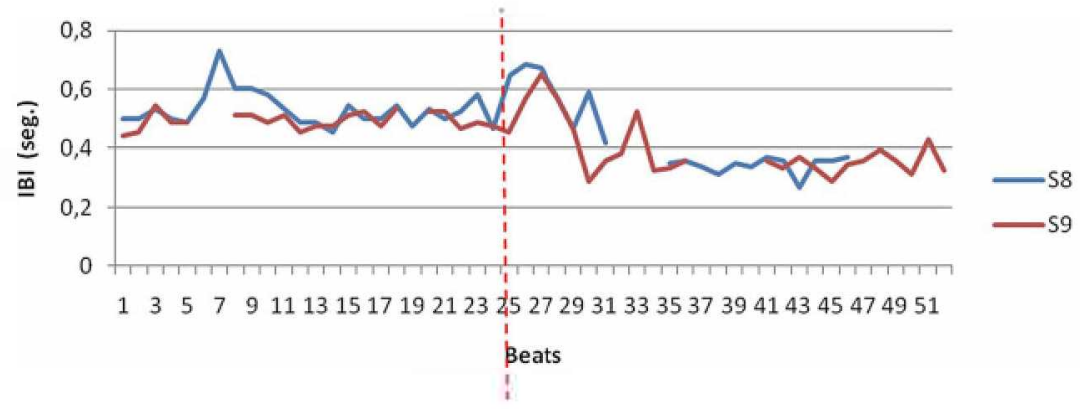

Figura 7. Medición del IBI en S8 y S9, la línea de puntos roja indica el OC (120-171 bpm)

Puede observarse además cómo en los casos de S2, S6 y S8, que venían produciendo variaciones del IBI anteriores al OC, la tendencia fue a continuar manteniendo el tempo anterior (120 bpm). En cambio, en los improvisadores que venían realizando pocas variaciones antes del cambio se observó en general una interrupción de la acción, la evaluación y el ajuste posterior. Este fue el caso para S1, S3 y S4. 


\section{Casos de entrainment en una proporción jerárquica o polirrímica}

El análisis de la performance desde la perspectiva del entrainment muestra cómo algunos improvisadores siguieron tocando en $120 \mathrm{bpm}$ luego del OC, incluso a lo largo de 10 beats (aproximadamente durante 4 seg). Esto no significa que no se adaptaran al cambio. Desde la perspectiva de la teoría del entrainment, en esta ejecución interactúan dos osciladores: (i) un oscilador humano (improvisador) a $120 \mathrm{bpm}$ aproximadamente y un oscilador mecánico (la base) a $171 \mathrm{bpm}$. Aunque esto sucede y el tempo del improvisador se mantiene a $120 \mathrm{bpm}$ aproximadamente, puede interpretarse que a pesar de ello, los osciladores se ajustan de alguna manera. Clayton $(2007,2012)$ ha descripto cómo en muchos casos ciertos ritmos se ajustan no solo al unísono sino también en una relación jerárquica (2:1, 4:2:1, 6:3:1) o polirrítmica (3:2, 4:3). Los resultados para S2 y $S 6$ muestran una organización temporal en el entrainment que puede interpretarse de acuerdo a este tipo de proporciones, a partir del OC. Detallamos a continuación lo ocurrido con S2 y S6.

S2: En este caso se observó un ajuste en una proporción 2:3, con un IBI promedio de $0,527 \mathrm{seg}$, valor correspondiente a un tresillo de negra en un tempo de $114 \mathrm{bpm}$. Es decir, en una proporción 2:3 con respecto a $171 \mathrm{bpm}$. Podría entenderse también que uno de cada tres beats correspondientes a la ejecución de S2, ajusta exactamente durante dos ciclos con uno de cada dos beats de la base. Luego hay un pequeño desajuste (marcado en la Figura 8 con una flecha oscura) y posteriormente un nuevo ciclo ajustado.

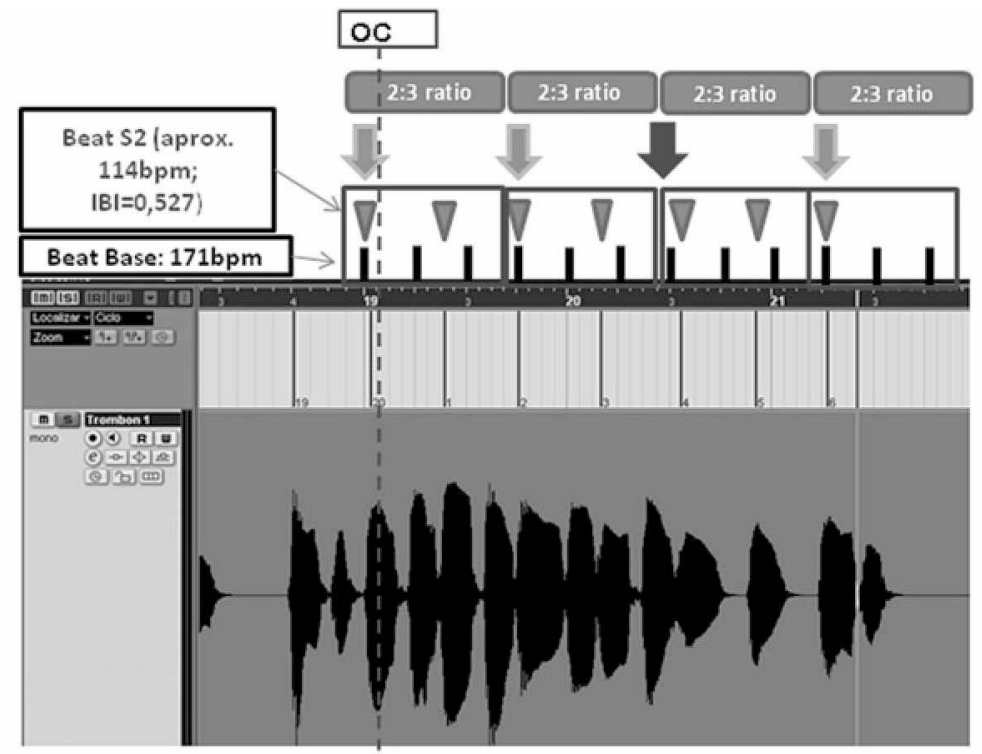

Figura 8. Tabla de medición del los IBI y captura de pantalla del Nuendo 4 para el S2, donde se observa la proporción 2:3.

Las flechas claras significan ajuste, las flechas oscuras desajuste. 
S6: Se observa una situación de ajuste en una proporción 2:3 en los primeros compases después de OC. Posteriormente se produce un desajuste y nuevamente un ajuste, pero en el último caso en una proporción 1:2. La situación de ajuste se señala en la Figura 9 con flechas claras, mientras que las oscuras indican un desajuste.

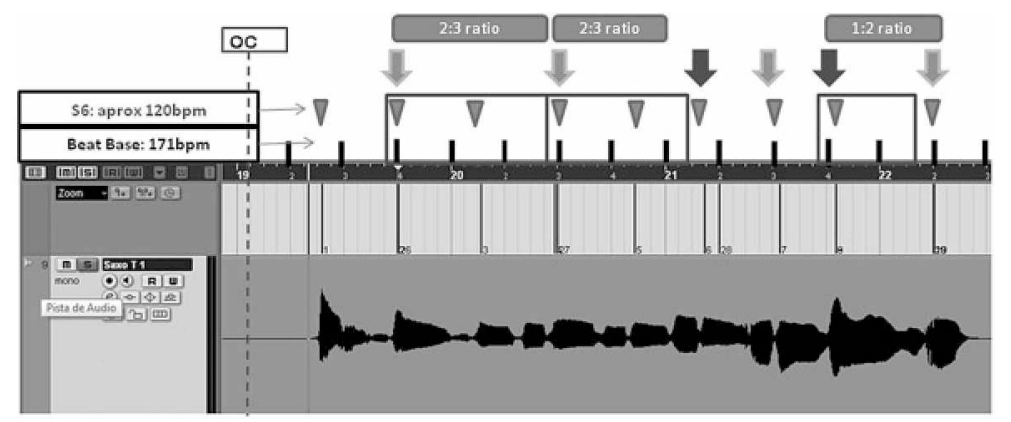

Figura 9. Tabla de medición del los IBI y captura de pantalla del Nuendo 4 para el S6, donde se observa la proporción 2:3.

Las flechas claras significan ajuste, las flechas oscuras desajuste.

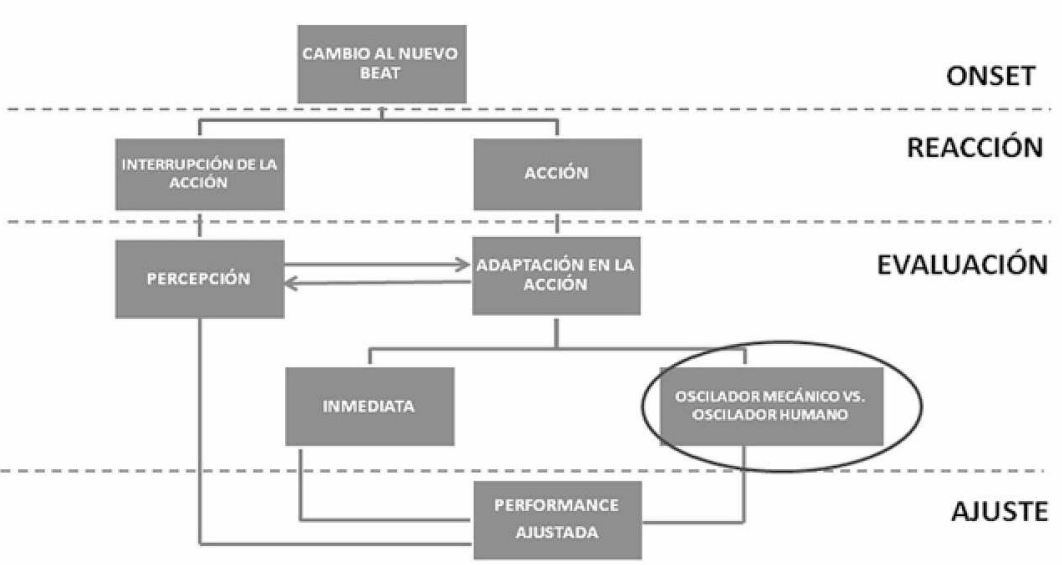

Figura 10. Modos en los que se produce el ajuste o adaptación al cambio.

A modo de síntesis del comportamiento general del grupo de improvisadores en el ciclo de percepción-acción, puede decirse que: (i) en todos los casos se produce una reacción, en la que el improvisador o bien sigue tocando, o bien interrumpe su acción para focalizar su percepción en la base; (ii) después de la reacción señalada en (i), en cualquiera de los dos casos el improvisador ingresa a una etapa de evaluación en la que se adapta en la acción, tocando o sin tocar, y focaliza su atención en la base; (iii) en los casos en los que el improvisador sigue tocando pueden suceder dos cosas: (iiia) que la adaptación sea inmediata o (iiib) que continúe con su ejecución de acuerdo a la sensación de tempo anterior (120 bpm) incluso hasta unos pocos segundos después del OC. Desde el punto de vista de la teoría del entrainment, podría interpretarse esta 
situación como un caso en donde el improvisador entra en sincronía con la base luego de un período de transición. Este período puede considerarse también como de evaluación; (iv) después de terminado el período de evaluación el oscilador humano entra en fase con el oscilador mecánico y se produce el ajuste (Figura 10).

\section{Análisis de los datos recolectados en la entrevista}

Luego de la Prueba 1 se realizaron dos preguntas breves a cada uno de los sujetos, en relación al cambio y su acción de ejecución con posterioridad al cambio. En las respuestas pudo observarse que:

(i) - Todos los improvisadores percibieron el OC en algún momento de su ejecución. El cambio fue descripto en términos de: cambio en el ritmo, en el pulso, en el tempo, y en la métrica. Si bien lo que cambiaba en la base era precisamente la velocidad del beat, el cambio fue evaluado a veces holísticamente en relación a la métrica o al ritmo en general.

(ii) - Las apreciaciones personales acerca de cómo respondieron al cambio incluyeron muchas de las acciones antes analizadas: "...acomodarme a la nueva velocidad" (S2); “...jugar con el ritmo para que quede más o menos acorde a lo que estaba sonando." (S3); "Sorpresa, un instante de confusión, adaptarme rápidamente al tempo."(S4); "Escuchar y seguir los cambios de tempo." (S5); "Parar, escuchar y tocar." (S6).

Al comparar el análisis de los resultados de la ejecución con las respuestas verbales se observó que éstas últimas no coinciden necesariamente con las acciones de ejecución efectivamente realizadas. Si bien los comentarios personales describen algunas de las acciones como, por ejemplo, seguir tocando, ajustarse a la base o parar de tocar, entre otras no existen referencias temporalmente más precisas que puedan brindar indicios de una percatación consciente de aspectos del ajuste en la performance como las que refieren a la proporcionalidad en el modo en que se realiza el ajuste (esto es, sincronía interactiva en una proporción 2:3 por ejemplo).

\section{Parte 3}

\section{Discusión y Conclusiones}

Los análisis realizados constituyen una pequeña muestra de los modos en que puede manifestarse temporalmente la interacción de los improvisadores sincronizando con una base. Las características identificadas 
ilustran algunas de las múltiples formas en las que los mecanismos de percepción-acción intervienen configurando la improvisación en la mente y el cuerpo del ejecutante. La reconfiguración de la acción por parte del improvisador, compromete la intervención de procesos de reacción, evaluación y ajuste, a partir de los cambios o modificaciones que ocurren frecuentemente en la ejecución de conjunto. Dichas modificaciones pueden definirse a priori como restricciones o limitantes temporales, formales, estilísticos, motívicas, etc. con las que los músicos, consciente o inconscientemente interactúan regulando constantemente su acción performativa. En el caso particular del estudio reportado se escogió la limitante temporal y se la puso en acción en una situación controlada de laboratorio para indagar aspectos de la acción adaptativa de cada ejecutante.

Dentro de este proceso adaptativo sería posible distinguir entre aquellos momentos en los que el improvisador está más atento a su propia performance, donde la concertación se daría en automático, y aquellos otros donde el músico está más atento a la percepción de lo que sucede en la base. En relación a esto, en el transcurso de la ejecución el improvisador realiza silencios no solo por una necesidad constructiva del discurso musical que está elaborando en tiempo real, sino también por la necesidad de percibir atentamente qué ocurre en la base.

Una vez introducido el cambio de tempo en la base, se produce una reconfiguración de la acción por parte de cada uno de los sujetos. En relación al momento de reacción, puede señalarse que ésta es instantánea y que de ahí en adelante el improvisador ingresa en un período de evaluación. El entrainment entre el improvisador y la base supone un proceso dinámico de continua adaptación que ya ha sido descripto en términos de groove en investigaciones anteriores (Doffman, 2008). Antes del cambio abrupto de tempo, ya existe un proceso de ajuste dinámico que viene sucediendo entre el sujeto y la base de acompañamiento. Este proceso es "experimentalmente intervenido" con la generación del dispositivo OC. Es por ello que el dispositivo nos permite el estudio del problema vinculado al foco atencional del músico. Luego de la reacción inicial ante el OC, el improvisador quiere entrar nuevamente en groove y al hacer esto reconfigura su acción. Esta reconfiguración de la acción, consciente o inconcientemente constituye, en términos de entrainment, una parte de la percatación consciente que integra la evaluación, en tanto ontología orientada por la percepción-acción en la ejecución improvisada.

Se considera que hay evaluaciones más o menos conscientes, dependiendo de si el improvisador está tocando o solo escuchando. La conciencia de la evaluación estaría determinada por los niveles temporales en la respuesta, a saber: 
(i) - un nivel temporal micro o muy corto (beat a beat); este nivel no puede ser experimentado en un nivel consciente;

(ii) - un nivel temporal medio (el improvisador interactúa tratando de ajustarse a un beat interno y/o externo); el improvisador realiza evaluaciones sobre el modo en el que viene produciéndose el ajuste en términos de groove;

(iii) - un nivel temporal macro donde existe una evaluación consciente (el improvisador decide si debe realizar una acción o si debe centrarse en alguno de los aspectos de la base).

La experiencia corporeizada del improvisador en la propia performance crea una "conciencia en acto" de la temporalidad en la improvisación. Esta conciencia implica la percatación consciente, tanto de la acción como del resultado de la acción, que se producen al mismo tiempo. Puede señalarse que aunque existe interacción en el nivel temporal micro, solo podría haber un tipo de reflexión consciente en un nivel temporal medio o más general. Es decir, que el improvisador podría desarrollar un tipo de evaluación holística acerca de su ajuste o desajuste en relación al groove, pero no llevar un tipo de control momento a momento, esto es, en el nivel nota a nota, o beat a beat. La posibilidad de reconfigurar la acción, estaría dada a partir del cambio de foco atencional desde el momento en el que el improvisador dirige su atención sobre lo percibido; tanto en relación a su propia ejecución, cuando esta se da "en automático"; o en relación a la base, cuando se producen detenciones en la acción. Los sucesos inesperados que alteran el modo en el que se viene produciendo el ajuste entre el improvisador y la base, modifican su atención generando de esta manera cambios de dirección en la producción del discurso musical. Es por esto último, que puede pensarse al proceso de interacción en la improvisación como un proceso de mutua adaptación, en el que la acción de cada uno de los músicos restringe y modifica las posibilidades de acción del otro.

La música improvisada es, en estos términos y en primer lugar, el producto emergente de un conjunto de interacciones entre los músicos al interior de un grupo musical. Se espera en futuros trabajos ampliar este estudio al análisis específico de la música improvisada como producto emergente del fenómeno intersubjetivo en el marco de un experimento que involucre a dos o más improvisadores. 


\section{Referencias}

Berliner, P. (1994). Thinking in Jazz: The infinite art of improvisation. Chicago: University of Chicago Press.

Clarke, E. (2005). Ways of listening: An ecological approach to the perception of musical meaning. Oxford: Oxford University Press.

Clayton, M., Sager, R., \& Will, U. (2005). In time with the music: The concept of entrainment and its significance for ethnomusicology. European Meetings in Ethnomusicology, 11, 3-142. Extraído de http://oro.open. ac.uk/2661/1/InTimeWithTheMusic.pdf.

Doffman, M. R. (2008). Feeling the groove: Shared time and its meanings for three jazz trios. (Ph.D. Dissertation - Music Department). Open University.

Huron, D. (2006). Sweet anticipation: Music and the psychology of expectation. Cambridge, Massachusetts: MIT Press.

Johnson, M. (2007). The meaning of the body: Aesthetics of human understanding. Chicago: The University of Chicago Press.

Leman, M. (2008). Embodied music cognition and mediation technology. Massachusettes: The MIT Press.

Meyer, L. B. (1956). Emotion and meaning in music. Chicago: University of Chicago Press.

Monson, I. (1996). Saying Something: Jazz improvisation and interaction. Chicago: The University of Chicago Press.

Pérez, J. B. (2013) Análisis de los aspectos armónicos en el ciclo de percepción-acción en la improvisación: Una propuesta empírica para el análisis con músicos de jazz argentinos. In D. Gonnet, M. I. Burcet \& R.

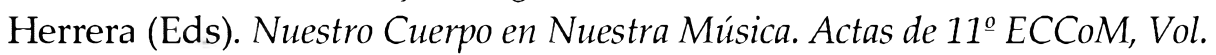
1, $N^{n} 1,241-251$. Buenos Aires: SACCoM.

Pérez, J. B., \& Martínez, I. C. (2012). Music improvisation as an embodied activity: The performer-envirorment interaction. Perspectives on Music Improvisation. Oxford (UK). 